\title{
A APLICAÇ̃̃ LOCALIZADA DE MONOAMÔNIO FOSFATO FAVORECE A DISPONIBILIDADE DE P NO SOLO E SUA ABSORÇÃO
}

\author{
Localized application of monoammonium phosphate favors the availability of $P$ in the soil and its absorption
}

Jaqueline Pereira Machado de Oliveira ${ }^{1}$, Luís Carlos Iuñes de Oliveira Filho ${ }^{1,}$ Elisandra Pocojeski

\author{
1. Professora; Departamento de Agronomia; Universidade do Oeste de Santa Catarina (UNOESC). E-mail: \\ jaqpmachado@gmail.com (*autor para correspondência) \\ 2. Universidade Tecnológica Federal do Paraná, Campus de Dois Vizinhos (UTFPR-DV), Coordenação da Engenharia Florestal \\ (COENF).E-mail: epocojeski@gmail.com
}

Artigo enviado em 24/01/2017, aceito em 16/03/2017 e publicado em 11/04/2017.

\begin{abstract}
RESUMO: O objetivo do presente trabalho foi avaliar a absorção de fósforo pelas plântulas de milho em função da aplicação localizada de fosfatos de cálcio e de amônio. O trabalho foi realizado em 2011, com amostra de um Cambissolo Húmico $(\mathrm{CH})$ e um Nitossolo Vermelho (NV). Os tratamentos consistiram da aplicação de superfosfato triplo (SFT), superfosfato simples (SS), diamônio fosfato (DAP) e monoamônio fosfato (MAP), além de uma

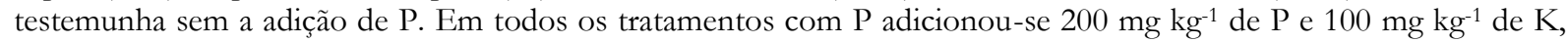
na forma de KCl; a quantidade de $\mathrm{N}$ adicionada pelo DAP $\left(160 \mathrm{mg} \mathrm{kg}^{-1}\right)$ foi ajustada nos demais tratamentos pela adição de $\mathrm{CaNO}_{3}$. A produção de massa seca da parte aérea (MSPA) foi maior nos tratamentos com SFT, SS e MAP, nas quais os incrementos foram respectivamente 71,67 e $62 \%$ maiores que a testemunha; enquanto com o DAP foi de $38 \%$. Os teores de P na parte aérea e nas raízes foram maiores no solo tratado com MAP. O SFT, MAP e DAP aumentaram as concentrações de $\mathrm{P}$ no solo, cuja média foi $93 \%$ superior a testemunha. A disponibilidade de $\mathrm{P}$ no solo e sua absorção são aumentadas pela aplicação localizada de MAP; por outro lado, a aplicação localizada de DAP inibe o crescimento de raízes.
\end{abstract}

Palavras-Chave - Fontes de fósforo. Liberação de nutrientes. Localização do fertilizante. Milho.

Abstract - The objective of this study was to evaluate the phorphorus absorption by corn seedlings according to the localized application of calcium and ammonium phosphates. The study was conducted in 2011, with samples of Inceptisol (CH) and Udox (NV). The treatments consisted on the application of triple superphosphate (TSP), simple superphosphate (SS) and diammonium phosphate (DAP) and monoammonium phosphate (MAP), beside a control without the addition of P. In all treatments with $\mathrm{P}$, were added $200 \mathrm{mg} \mathrm{kg}^{-1}$ of $\mathrm{P}$ and $100 \mathrm{mg} \mathrm{kg}^{-1}$ of K as KCl; the amount of $\mathrm{N}$ added by DAP $\left(160 \mathrm{mg} \mathrm{kg}^{-1}\right)$ was adjusted in all treatments by adding $\mathrm{CaNO}_{3}$. Dry matter production of the aerial part (MSPA) was higher in treatments with STF, SS and MAP, in which were respectively $71,67,62 \%$ higher than the control; while with the DAP was $38 \%$. The phosporus content in the shoots and roots were higher in the soil treated with MAP. The TSP, MAP, and DAP increased P concentrations in the soil, an average $93 \%$ higher than the control. The availability of $\mathrm{P}$ in the soil and its absorption in increased by localized application of MAP; however, localized application of DAP, inhibits the growth of roots.

Keywords - Sources of phosphorus. Nutrient release. Location of the fertilizer. Corn

\section{INTRODUÇÃO}

O milho em termos de cultivo e consumo é um dos cereais mais importantes no mundo, com projeções de produção e consumo nacional em 2014/15 de, aproximadamente, 62 e 51 mil toneladas, respectivamente (MAPA, 2013). Assim como a maioria dos cereais, esta cultura apresenta altas produtividades quando aplicadas quantidades suficientes de fósforo em solos com deficiência deste nutriente. Isto ocorre porque o milho necessita de $\mathrm{P}$ para a formação e desenvolvimento de seus grãos e 
espigas e sua falta pode limitar a produtividade (LUCENA et al., 2000).

A elevada acidez e a baixa disponibilidade de fósforo encontradas nos solos da região Sul do país requerem a adição de corretivo de acidez e de fontes fosfatadas para obtenção de melhores rendimentos vegetais. Porém, a eficácia do fósforo adicionado ao solo varia dentre outros: com o tipo de fertilizante fosfatado (CORRÊA et al., 2008), com o método de aplicação (COSTA et al., 2009), e com a quantidade aplicada (RESENDE et al., 2006), além de variar com atributos do solo, como pH (VIÉGAS et al., 2010), umidade (RANNO et al., 2007), teor de argila (SIMÕES NETO et al., 2015), teor de Ca (BROGGI et al., 2010) e formas de Fe (FINK et al., 2014).

A aplicação localizada de fósforo diminui o volume de solo fertilizado com o qual as raízes entram em contato, podendo prejudicar a absorção do nutriente, especialmente em períodos de déficit hídrico. Segundo Havlin et al., (2005), à medida que aumenta o contato fertilizante-raiz, diminiu sua sorção pelo solo. Barber (1995) relatou ainda que a aplicação localizada dos fertilizantes fosfatados na cultura do milho promove maior desenvolvimento radicular nestas regiões, e que a quantidade aplicada ao solo, assim como seu nível inicial influenciam no grau de proliferação deste sistema.

Com relação às fontes solúveis, os fertilizantes fosfatados diferem entre si, principalmente na sua composição química, na sua solubilidade e em seu cátion acompanhante. Estas diferenças podem influenciar a taxa de solubilização dos fosfatos e as suas reações, especialmente de precipitação e adsorção química de fósforo nas regiões próximas aos grânulos ou partículas de fosfato, cuja intensidade é alta. Isto irá refletir na concentração de $\mathrm{P}$ na solução do solo e poderá influenciar a eficiência de utilização deste nutriente pelas plantas e, consequentemente o seu rendimento (ROTILI et al., 2010).

É das microrregiões ao redor dos grânulos ou de suas proximidades, que as raízes absorvem os nutrientes aplicados, entretanto, são raros os trabalhos visando à aplicação localizada de diferentes fontes fosfatadas. Neste sentido, objetivou-se avaliar a absorção de $\mathrm{P}$ pelas plântulas de milho e seu valor remanescente, sob efeito da aplicação localizada de diferentes fertilizantes fosfatados.

\section{MATERIAL E MÉTODOS}

O experimento foi realizado em casa de vegetação, na faculdade de Agronomia da
Universidade do Estado de Santa Catarina (UDESC), em Lages, SC, em 2011. Utilizaram-se amostras de um Cambissolo Húmico $(\mathrm{CH})$ e um Nitossolo Vermelho (NV), nas quais apresentaram as seguintes características químicas, na camada de $0-20 \mathrm{~cm}$ : pH(água): 4,5 e 4,6; 7,3 e 7,1 $\mathrm{mg} \mathrm{dm}^{-3}$ de P; 1,8 e 0,6 $\mathrm{cmol}_{\mathrm{c}} \mathrm{dm}^{-3}$ de Ca; CTC $(\mathrm{pH} 7,0)$ de 30,1 e 20,3 cmol $_{\mathrm{c}} \mathrm{dm}^{-}$ 3, 400 e $790 \mathrm{~g} \mathrm{~kg}^{-1}$ de argila, além de 50 e $23 \mathrm{~g} \mathrm{dm}^{-3} \mathrm{de}$ matéria orgânica (MO), respectivamente.

As unidades experimentais foram constituídas por colunas de PVC com $10 \mathrm{~cm}$ de diâmetro e $12 \mathrm{~cm}$ de comprimento, preenchidas com $1,10 \mathrm{~kg}$ de solo (base seca). Cada unidade experimental foi constituída pela justaposição de anéis concêntricos, sendo um com $2 \mathrm{~cm}$ de espessura, localizado na extremidade superior, seguido de cinco outros com $1 \mathrm{~cm}$ de espessura cada e de um último com $5 \mathrm{~cm}$ de espessura, localizado na extremidade inferior.

Os tratamentos consistiram da adição de superfosfato triplo (SFT), superfosfato simples (SS), diamônio fosfato (DAP) e monoamônio fosfato (MAP), todos na dose de $200 \mathrm{mg} \mathrm{kg}^{-1}$ de P (916 kg ha${ }^{1}$ de $\mathrm{P}_{2} \mathrm{O}_{5}$ ), além de uma testemunha sem a adição de P. Em todos os tratamentos adicionou-se $100 \mathrm{mg} \mathrm{kg}^{-1}$ de $\mathrm{K}$, na forma de $\mathrm{KCl}$; e a quantidade de $\mathrm{N}$ adicionada pelo DAP (160 $\mathrm{mg} \mathrm{kg}^{-1}$ ) foi ajustada em todos os demais tratamentos pela adição de $\mathrm{CaNO}_{3}$. As doses de $\mathrm{P}$ e $\mathrm{K}$ foram determinadas com base nos resultados do outro estudo (OLIVEIRA et al., 2014). Antes da aplicação dos tratamentos, a acidez dos solos foi corrigida pela adição de calcário dolomítico para elevar o $\mathrm{pH}$ a 6,0, no qual foi aplicado 15 dias antes da montagem do experimento. Os grânulos de cada fertilizante (SFT, SS, DAP, MAP e KCl) tinham diâmetro entre 0,5 e $2 \mathrm{~mm}$. O $\mathrm{CaNO}_{3}$ e o $\mathrm{KCl}$ foram distribuídos uniformemente em todo o solo da coluna e os fertilizantes fosfatados foram aplicados somente no solo que compreendeu a espessura do terceiro anel, ou seja, na camada $2-3 \mathrm{~cm}$ da coluna. Isto ocorreu para que o fósforo ficasse mais próximo a semente.

Os solos foram umedecidos até a capacidade de campo antes de serem colocados nas colunas, cuja umidade foi reposta diariamente ao longo de todo o período experimental. Cultivaram-se três plantas de milho (Codetec 35) por unidade experimental, durante 20 dias após a emergência. Ao final do período experimental colheu-se a parte aérea e separaram-se as raízes do solo, estes foram secos e, após, determinaram-se a massa seca de parte aérea (MSPA) e de raízes (MSR). Posteriormente a colheita, o solo de todas as camadas da coluna foi 
homogeneizado, retirado uma amostra e acondicionado em estufa.

No tecido vegetal, nas raízes e no solo foram determinados os valores de $\mathrm{P}$ disponível e $\mathrm{Ca}$, de acordo com metodologia proposta por Tedesco et al. (1995). Utilizou-se o delineamento experimental inteiramente casualizado, com quatro repetições.

Os resultados obtidos foram avaliados estatisticamente por meio de análise da variância utilizando-se o teste de Tukey $(\mathrm{P}<0,05)$.
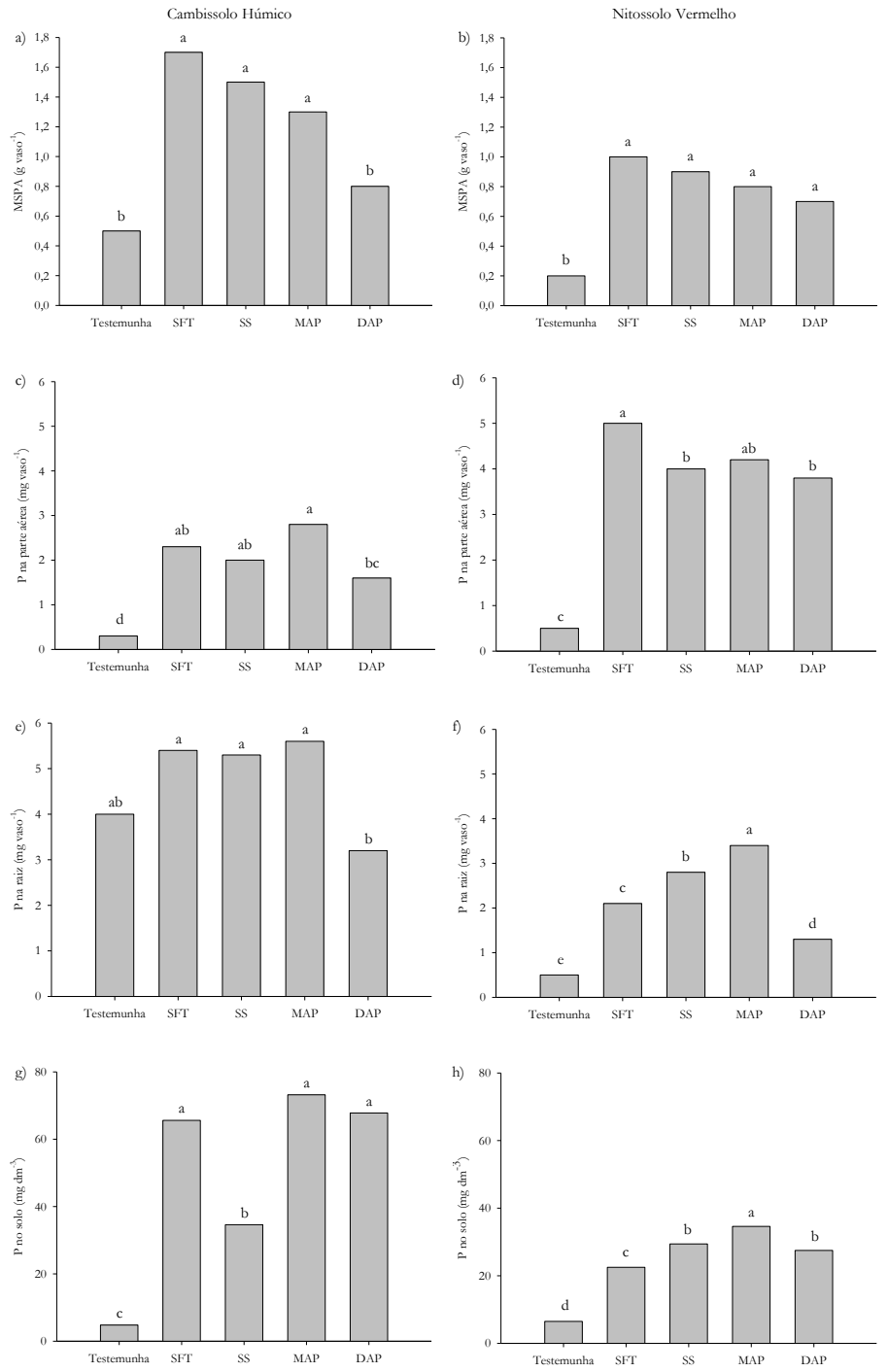

Figura 1. Massa seca da parte aérea (MSPA), teores de $\mathbf{P}$ na parte aérea e raiz de plântulas de milho e concentração de $\mathbf{P}$ no solo, em função da adição de adubos fosfatados (supersfosfato triplo, SFT; superfosfato simples, SS; diamônio fosfato, DAP e monoamônio fosfato, MAP) em dois solos catarinenses. Médias seguidas de letras distintas diferem os tratamentos significativamente entre si pelo teste de Tukey a $5 \% *_{\text {ns }}=$ não houve diferença estatística entre os tratamentos. 
A resposta do milho à adubação fosfatada provavelmente ocorreu devido à elevada necessidade de $\mathrm{P}$ requerida por esta cultura para o seu crescimento inicial, indicando a liberação rápida do nutriente das fontes solúveis, proporcionando maior incremento na MSPA comparado ao tratamento sem adubo. Este resultado também foi observado em um estudo com plantas de milho, onde maiores rendimentos foram atingidos no primeiro cultivo quando se utilizou fosfato solúvel no plantio comparado a fosfato natural (ONO et al., 2009).

A menor produção de MSPA observada no $\mathrm{CH}$ utilizando DAP se deve, provavelmente, ao efeito tóxico promovido por esse adubo, no qual possui $\mathrm{NH}_{4}{ }^{+}$em sua composição comparado aos demais tratamentos (onde o $\mathrm{N}$ foi adicionado na forma de $\left.\mathrm{CaNO}_{3}\right)(\mathrm{OLIVEIRA}$ et al, 2003). Além disto, o DAP possui maior índice salino dentre as fontes fosfatadas aplicadas, fator que pode ter promovido uma desidratação na planta e prejudicado a produção de raízes e, consequentemente, a produção de MSPA (NOVAIS et al, 2007).

Há relatos que a adubação localizada de fósforo proporciona efeitos negativos sobre a produtividade, o que pode ser atribuído à salinidade, na qual afetariam a germinação de sementes e o crescimento de raízes (PERYEA, 1990). Barreto e Fernandes (2002) verificaram que plantas de milho adubadas com SFT a lanço obtiveram produtividades maiores comparadas com adubações no sulco de plantio, sendo essa diferença atribuída a possível salinidade ou toxidez pela aplicação localizada da fonte de P.

Os tratamentos diferiram quanto à produção de massa seca de raiz (MSR) somente no CH (Figura 2a). A produção de MSR em todos os tratamentos variou de 2,1 a 1,1 g vaso-1, sendo maiores nos tratamentos com SS, T, SFT e MAP comparadas ao DAP. A diferença entre a média dos tratamentos com maior produção (SS, SFT, T e MAP) e o tratamento com DAP foi de $44 \%$.

A maior produção de MSR observada na testemunha pode ser devida à distribuição da energia de assimilação dos produtos da fotossíntese, que são direcionados para a formação e desenvolvimento do sistema radicular quando a concentração do nutriente é baixa, visando aumentar a sua área de absorção. Quando alguns nutrientes encontrarem-se limitando o crescimento das plantas, especialmente $\mathrm{P}$ e $\mathrm{N}$, as raízes tornam-se os drenos preferenciais de fotoassimilados, podendo ser uma estratégia de adaptação desenvolvida para aumentar a eficiência de absorção (HORST et al., 1993).

A ineficiência do DAP na produção de de MSR nas plântulas de milho relativamente aos demais fosfatos provavelmente está relacionada com a presença de amônio na composição deste fertilizante criando uma barreira tóxica que pode ter inibido o crescimento e o desenvolvimento das raízes comparado aos demais tratamentos que receberam $\mathrm{CaNO}_{3}$ como fonte de $\mathrm{N}$.

Os tratamentos contendo fontes fosfatadas proporcionaram aumento nos teores de $\mathrm{P}$ na parte aérea, em ambos os solos (Figura 1c e 1d). No CH, o MAP destacou-se com maior incremento, atingindo $2,8 \mathrm{mg}$ vaso $^{-1}$, ou seja, um valor $89 \%$ maior que a testemunha. No outro solo, o maior teor de $\mathrm{P}$ na parte aérea foi encontrado onde aplicou-se SFT, atingindo $5,0 \mathrm{mg} /$ vaso. Os demais tratamentos com fosfato variaram entre 3,8 a $4,2 \mathrm{mg} \mathrm{vaso}^{-1}$; e a testemunha apresentou o menor teor, igual a $0,5 \mathrm{mg}$ vaso $^{-1}$. No entanto, todas as fontes aplicadas ficaram dentro da faixa de suficiência para esta cultura que é de 1,0 a $1,5 \mathrm{~g} \mathrm{~kg}^{-1}$ (FURLANI, 2004).

Os maiores valores de $\mathrm{P}$ na parte aérea nos tratamentos com MAP e SFT para $\mathrm{CH}$ e NV, respectivamente, podem ter ocorrido porque o MAP age no solo alterando o $\mathrm{pH}$ ao redor de seus grânulos de maneira semelhante ao SFT, ou seja, nos períodos subsequentes a sua aplicação ao solo ele diminui o pH devido ao consumo de $\mathrm{H}^{+}$pela transformação de parte das moléculas de $\mathrm{HPO}_{4}^{-2}$ em $\mathrm{H}_{2} \mathrm{PO}_{4}^{-}$, em função do equilíbrio existente entre essas duas formas. Isto favorece a dissolução de fosfatos que estejam ligados ao $\mathrm{Ca}$, podendo aumentar assim a disponibilidade de P (ERNANI, 2008).

Os teores de $\mathrm{P}$ nas raízes das plântulas de milho variaram com os tratamentos, em ambos os solos (Figura 1e e 1f). No $\mathrm{CH}$, os tratamentos T, MAP, SFT e SS apresentaram teores que variaram de 4,0 a $5,6 \mathrm{mg} \mathrm{vaso}{ }^{-1}$. Eles foram maiores que o DAP, que apresentou valor igual a $3,2 \mathrm{mg}$ vaso $^{-1}$. Para o $\mathrm{NV}$, o maior teor de $\mathrm{P}$ na raiz foi obtido no MAP $(3,4$ $\mathrm{mg}$ vaso $\left.^{-1}\right)$; já o DAP apresentou o menor valor dentre as fontes de P. A diferença entre o MAP e a T foi de $85 \%$. O efeito superior do tratamento com MAP comparado ao DAP, nos leva a crer que o diamônio fosfato favoreceu uma maior precipitação do $\mathrm{P}$ (com o $\mathrm{Ca}$ ) ao redor de seus grânulos comparado aos demais tratamentos e com isto diminuiu a sua disponibilidade e absorção, adicionalmente, há o efeito tóxico do DAP (NOVAIS et al., 2007).

As concentrações de P no solo aumentaram 
em todos os tratamentos, nos dois solos (Figura $1 \mathrm{~g}$ e 1h). No CH, o MAP, o DAP e o SFT foram os tratamentos que proporcionaram as maiores concentrações de $\mathrm{P}$ no solo, com valores respectivamente iguais a $73 ; 68$ e $66 \mathrm{mg} \mathrm{dm}^{-3}$; o tratamento com SS apresentou concentração inferior aos anteriores. A diferença entre a $\mathrm{T}$ e a média dos tratamentos com MAP, DAP e SFT foi de 93\%. No $\mathrm{NV}$, assim como ocorreu com as raízes, a maior concentração de P no solo foi igual a $34,6 \mathrm{mg} \mathrm{dm}^{-3}$ no tratamento com MAP. Os demais tratamentos foram menores que o MAP e diferentes entre si, com valores variando de 6,5 a $29,4 \mathrm{mg} \mathrm{dm}^{-3}$.
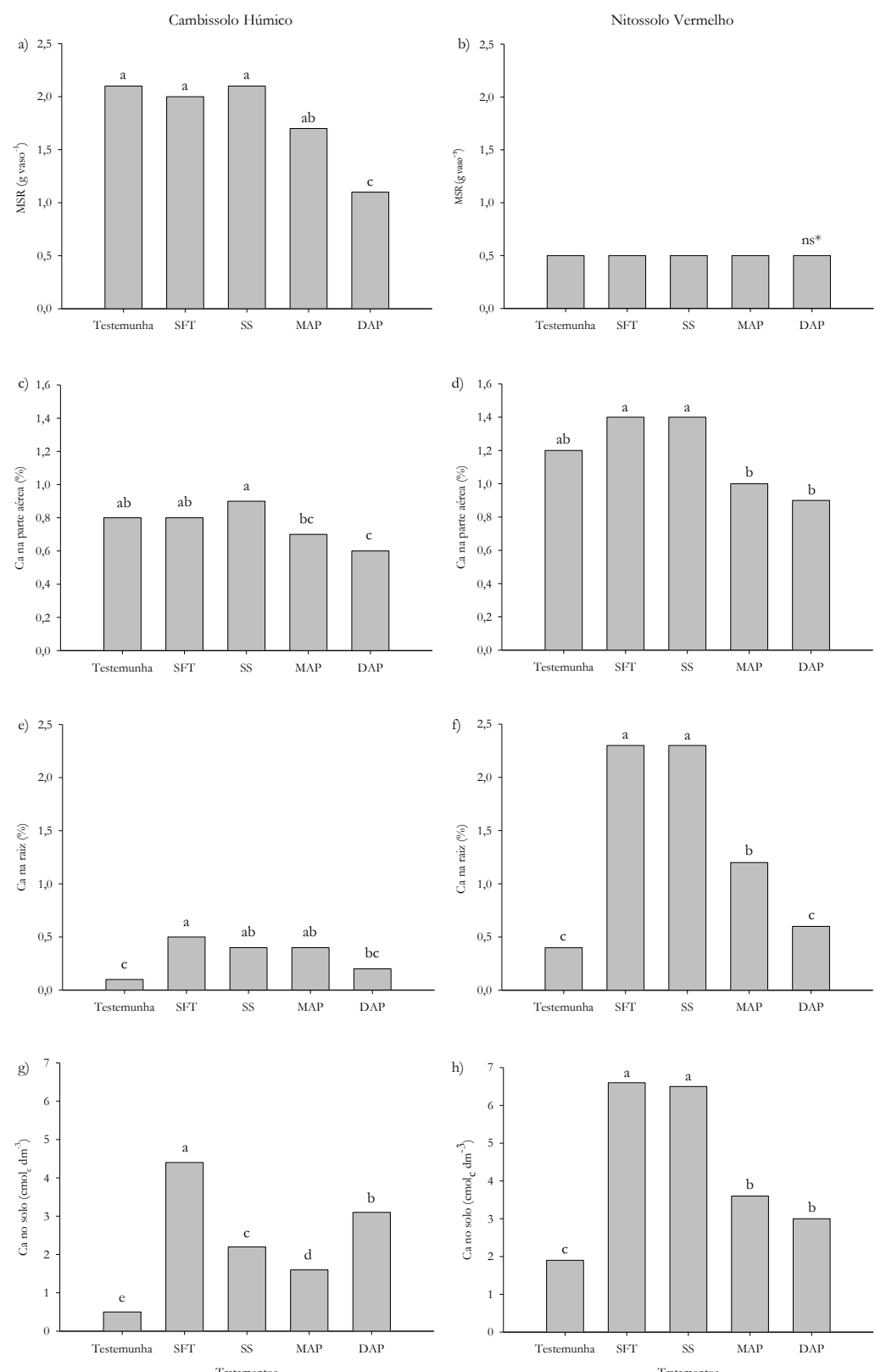

Figura 2. Massa seca de raiz (MSR), teores de Ca na parte aérea e raiz de plântulas de milho e concentração de Ca no solo, em função da adição de adubos fosfatados (supersfosfato triplo, SFT; superfosfato simples, SS; diamônio fosfato, DAP e monoamônio fosfato, MAP) em dois solos catarinenses. Médias seguidas de letras distintas diferem os tratamentos significativamente entre si pelo teste de Tukey a $5 \%$. *ns= não houve diferença estatística entre os tratamentos. 
Exceto na $\mathrm{T}$, as quantidades de $\mathrm{P}$ disponíveis presentes nos solos após o cultivo de milho, com relação ao fósforo aplicado no início do experimento variaram de 35 a $73 \mathrm{mg} \mathrm{dm}^{-3}$. Estes valores equivaleram a 17,3 e $36,6 \%$ do $\mathrm{P}$ aplicado. Isto nos permite inferir que, a dose aplicada ao solo foi pouco aproveitada pela cultura, fato que geralmente ocorre em nossos solos (extremamente intemperizados), no qual a maior parte do $\mathrm{P}$ adicionado é adsorvido especificamente por óxidos de $\mathrm{Fe}, \mathrm{Al}$ e $\mathrm{Mn}$, nas arestas das argilas silicatadas e nos aluminossilicatos amorfos, além de ter a possibilidade de formar precipitados. Segundo Parfitt (1989), a adsorção de $\mathrm{P}$ ocorre em três estádios e na forma binucleada, conforme o tempo de reação: no primeiro, a reação dá-se rápida e fortemente em sítios muito reativos, onde silicatos, $\mathrm{OH}^{-} \mathrm{e} \mathrm{OH}_{2}{ }^{+}$, são trocados por fosfato; no segundo, ele é adsorvido em sítios menos reativos e, no terceiro, migra via porosidade para os lóbulos internos dos cristais, podendo ser considerado como precipitação.

Nos dois solos, os teores de Ca da parte aérea foram afetados pelos tratamentos (Figura 2c e 2d). O maior teor de $\mathrm{Ca}$ foi encontrado no SS, no entanto, não diferiu do SFT e da T. O MAP e DAP não promoveram aumentos nesta variável. $\mathrm{O}$ cálcio presente no tecido variou de 0,9 a $0,6 \%$ nos tratamentos. No NV, a $\mathrm{T}$ foi igual aos fosfatos de cálcio e de amônio, no entanto, os teores de Ca na parte aérea foram maiores nas fontes de cálcio comparados as de amônio. A magnitude desta variável, neste solo, foi de 0,9 a $1,4 \%$. Os valores encontrados em todos os tratamentos estão dentro da faixa de suficiência que é de 0,2 a $0,8 \%$ para a cultura do milho (FURLANI, 2004).

Os maiores teores de $\mathrm{Ca}$ na parte aérea proporcionados pelas fontes de fosfato de cálcio se deve à presença deste nutriente na fórmula do SS e SFT, que apresentam aproximadamente $18 \%$ e $15 \%$ de $\mathrm{Ca}$, respectivamente. No entanto, pode-se observar que os valores não variaram comparados à testemunha, o que pode ser explicado pelo pouco tempo de cultivo (20 dias).

Nas raízes, com exceção do DAP, todos os tratamentos promoveram aumentos nos teores de $\mathrm{Ca}$, em ambos os solos (Figura 2e e 2f). No $\mathrm{CH}$, o maior incremento foi proporcionado pelo SFT na qual atingiu $0,5 \%$ de Ca. Entretanto, a variação nos demais tratamentos foi pequena de 0,1 a $0,4 \%$. No NV, os valores foram maiores nos tratamentos com SS e SFT, com valor igual a 2,3\% para ambos; já no MAP o teor atingiu $1,2 \%$, decrescendo no DAP e T.
Em ambos os solos, a adição de fontes de $\mathrm{P}$ aumentaram as concentrações de $\mathrm{Ca}$ neste meio (Figura $2 \mathrm{~g}$ e $2 \mathrm{~h}$ ). No $\mathrm{CH}$ destacamos o SFT com o maior incremento, seguido pelo DAP, SS e MAP. Os valores encontrados para SFT, DAP, SS e MAP variaram de 4,4 a 1,6 $\mathrm{cmol}_{\mathrm{c}} \mathrm{dm}^{-3}$, sendo que a diferença entre o SFT e a T foi de $89 \%$. No NV, os fosfatos de cálcio e os fosfatos de amônio aumentaram os teores de Ca no solo em média 71 e $42 \%$ comparados a $\mathrm{T}$, respectivamente.

Os maiores teores de $\mathrm{Ca}$ obtidos nos tratamentos que receberam SFT pode ser influência da presença deste nutriente na sua composição que proporciona maiores concentrações de cálcio em solução e, consequentemente, maior absorção. Mantovani et al. (2007) observaram que a aplicação combinada de $\mathrm{CaNO}_{3}$ com SFT aumentou a lixiviação de cálcio em um Nitossolo Vermelho. Porém, no presente trabalho como o sistema é fechado, o Ca foi para a solução e consequentemente foi absorvido pela planta.

\section{CONCLUSÕES}

A adição localizada do fertilizante monoamônio fosfato ao solo favorece o aumento da disponibilidade de P neste meio e, consequentemente, sua absorção pela planta, especialmente no $\mathrm{CH}$. A adição localizada de superfosfato triplo, superfosfato simples e monoamônio fosfato aumentam a produção de MSPA das plantas de milho, com destaque para o SFT; por outro lado o rendimento de raízes é inibido pela aplicação localizada de DAP.

\section{REFERÊNCIAS}

BARBER, S. A. Mecanismos de absorção de fósforo sob condições de estresse ambiental. In: SIMPÓSIO INTERNACIONAL SOBRE ESTRESSE AMBIENTAL, 1., Belo Horizonte. 1992, Anais... Sete Lagoas, Empresa Brasileira de Pesquisa Agropecuária, p. 233-237, 1995.

BARRETO, A.C.; FERNANDES, M.F. Produtividade e absorção de fósforo por plantas de milho em função de doses e modos de aplicação de adubo fosfatado em solo de tabuleiro costeiro. Revista Brasileira de Ciência do Solo, v.26, p.151-156, 2002.

BROGGI, F.; FREIRE, F. J.; FREIRE, M. B. G. S.; NASCIMENTO, C. W. A.; OLIVEIRA, A. C. 
Avaliação da disponibilidade, adsorção e níveis críticos de fósforo em diferentes solos. Revista Ceres, v. 57, n. 2, p. 247-252, 2010.

CORREAA, R. M.; NASCIMENTO, C. W. A.; FREIRE, F. J.; SOUZA, S. K. S.; SILVA, G. B. Disponibilidade e níveis críticos de fósforo em milho e solos fertilizados com fontes fosfatadas. Revista Brasileira de Ciências Agrárias, v. 3, n. 3, p. 218-224, 2008.

COSTA, S. E. V. G.; SOUZA, E. D.; ANGHINONI, I.; FLORES, J. P. C.; CAO, E. G.; HOLZSCHH, M. $\mathrm{J}$. Phosphorus and root distribution and corn growth as related to long-term tillage Systems and fertilizer placement. Revista Brasileira de Ciência do Solo, v. 33, p. 1237-1247, 2009 .

ERNANI, P. R. Química do solo e disponibilidade de micronutrientes. Lages, 2008. 230p.

FINK, J. R.; INDA, A. V. BAYER, C.; TORRENT, J.; BARRÓN, V. Mineralogy and phosphorus adsorption in soils of south and central-west Brazil under conventional and no-tillage systems. Acta Scientiarum Agronomy, v. 36, n. 3, p. 379-387, 2014.

FURLANI, A. M. C. Nutrição Mineral. In: KERBAUY, G. B. Fisiologia vegetal. Rio de Janeiro, Guanabara Koogan. 2004, p. 40-75.

HAVLIN, J. L.; BEATON, J. D.; TISDALE, S. L.; NELSON, W. L. Soil fertility and fertilizers. 7.ed. Nova York, Macmillan, 2005. 515p.

HORST, W. J.; ABDOU, M.; WIESLER, F. Genotypic differences in phosphorus efficiency of wheat. Plant and Soil, v. 155, p. 293-296, 1993.

LUCENA, L. F. C.; OLIVEIRA, F. A.; SILVA, I. F.; ANDRADE, A. P. Respostas do milho a diferentes níveis de nitrogênio e fósforo aplicados ao solo. Revista Brasileira de Engenharia Agrícola e Ambiental, v. 4, n. 3, p. 334-337, 2000.

MANTOVANI, A.; ERNANI, P. R.; SANGOI, L. A adição de superfosfato triplo e a percolação de nitrogênio no solo. Revista Brasileira de Ciência do Solo, v. 31, p. 887-895, 2007.

MAPA - MINISTÉRIO DA AGRICULTURA PECUÁRIA E ABASTECIMENTO. Projeções do Agronegócio Brasil, 2011/12 a 2021/22. 7p. 2013. http://www.agricultura.gov.br/arq editor/file/Minis terio/gestao/projecao/Tabelas $\% 20$ gerais.pdf. Acesso 19 JAN 2015

NOVAIS, R. F.; SMYTH, T. J.; NUNES, F. N. Fósforo. In: NOVAIS, R. F.; ALVAREZ V., V. H.; BARROS, N. F.; FONTES, R. L. F.; CANTARUTTI, R. B.; NEVES, J. C. L. Fertilidade do Solo. Viçosa, MG, Sociedade Brasileira de Ciência do Solo, 2007. p. 737-768.

OLIVEIRA, J. P. O.; ERNANI, P. R.; GATIBONI, L. C.; PEGORARO, A. Alterações químicas e avaliação de $\mathrm{P}$ disponível na região adjacente aos grânulos de superfosfato tripo e diamônio fosfato em solos ácidos. Revista Brasileira de Ciência do Solo, v. 38, p. 1526-1536, 2014.

OLIVEIRA, A. P.; PEREIRA, E. L.; BRUNO, R. L. A.; ALVES, E. U.; COSTA, R. F.; LEAL, F. R. F. Produção e qualidade fisiológica de sementes de feijão-vagem em função de fontes e doses de nitrogênio. Revista Brasileira de Sementes, v. 25, no 1 , p.49-55, 2003.

ONO, F. B.; MONTAGNA, J.; NOVELINO, J. O.; SERAFIM, M. E.; DALLASTAS, D. C.; GARBIATES, M. V. Eficiência agronômica de superfosfato triplo e fosfato natural de arad em cultivos sucessivos de soja e milho. Ciência Agrotécnica, v. 33, n. 3, p. 727-734, 2009.

PARFITT, R. L. Phosphate reactions with natural allophane, ferrihydrite and goethite. Journal Soil Science, v.40; p.359-369, 1989.

PERYEA, F. J. Phosphate: fertilizer: induced salt toxicity of newly planted apple trees. Soil Science Society American Journal, Madison, v. 54, n. 6, p. 1778-1783, 1990.

RANNO; S. K.; SILVA, L. S.; GATIBONI, L. C.; RHODEN, A. C. Capacidade de adsorção de fósforo em solos de várzea do estado do Rio Grande do Sul. Revista Brasileira de Ciência do Solo, v. 31, p. 21-28, 2007.

RESENDE, A. V.; FURTINI NETO, A. E.; ALVES, V. M. C.; MUNIZ, J. A.; CURI, N.; FAQUIN, V.; KINPARA, D. I.; SANTOS, J. Z. L.; CARNEIRO, L. F. Fontes e modos de aplicação de fósforo para o milho em solo cultivado da Região do Cerrado. Revista Brasileira de Ciência do Solo, v. 30, p. 453- 
466, 2006.

ROTILI, E. A.; FIDELIS, R. R.; SANTOS, M. M.; BARROS, H. B. PINTO, L. C. Eficiência do uso e resposta à aplicação de fósforo de cultivares de arroz em solos de terras altas. Bragantia, v. 69, n. 3, p. $705-$ 710, 2010.

SIMÕES NETO, D. E.; OLIVEIRA, A. C.; FREIRE, F. J.; FREIRE, M. B. G. S.; OLIVEIRA, E. C. A.; ROCHA, A. T. Adubação fosfatada para cana- $\mathrm{de}^{-}$acucar em solos representativos para $\mathrm{O}$ cultivo da espécie no Nordeste brasileiro. Pesquisa Agropecuária Brasileira, v. 50, n. 1, p. 73-81, 2015.

TEDESCO, M. J.; GIANELLO, C.; BISSANI, C. A.; BOHNEN, H.; VOLKWEISS, S. J. Análises de solo, plantas e outros materiais. $2^{a}$ ed. Porto Alegre: Universidade Federal do Rio Grande do Sul, 1995. (Boletim técnico, 5).

VIÉGAS, R. A.; NOVAIS, R. F.; SCHULTHAIS, F. Availability of a soluble phosphorus source applied to soil samples with different acidicity levels. Revista Brasileira de Ciência do Solo, v. 34, p. 1125-1136, 2010. 